\title{
Tropical ocean hydroclimate and temperature from coral archives
}

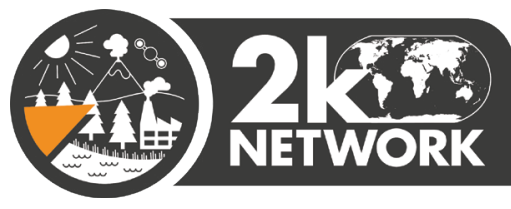

\author{
Jessica Hargreaves ${ }^{1}, 2$, K. DeLong ${ }^{3}$, T. Felis ${ }^{4}$, N. Abram ${ }^{1,2}$, K. Cobb ${ }^{5}$ and H. Sayani ${ }^{5}$ \\ 1st CoralHydro2k workshop, Sydney, Australia, 1 September 2019
}

The CoralHydro2k project of the PAGES $2 k$ Network met at the University of New South Wales before the 13th International Conference on Paleoceanography in Sydney, Australia, for our first in-person meeting (pastglobalchanges.org/calendar/2019/127pages/1744). The goal of CoralHydro2k is to build upon Ocean2k temperature reconstructions (Tierney et al. 2015) by using new and existing coral-based reconstructions to examine temperature and salinity changes (i.e. hydroclimate) through paired records of coral strontium to calcium ( $\mathrm{Sr} / \mathrm{Ca}$ ) and oxygen isotopic ratios $\left(\delta^{18} \mathrm{O}\right.$; e.g. Felis et al. 2018). The 24 scientists, with three participating remotely, included working group members, coral experts, and experts in other fields. The workshop started with a review of the aims of the project followed by updates of current temperature and salinity reconstructions, and concluded with further discussions about the best practices for coral-based reconstructions.

One aim of CoralHydro2k is the generation and inclusion of new paired coral $\mathrm{Sr} / \mathrm{Ca}$ and $\delta^{18} \mathrm{O}$ records that allow for investigations of seasonal-to-multidecadal variability of hydroclimate. The project incorporates these new records, along with those included in the Ocean2k dataset (PAGES2k Consortium 2017; Tierney et al. 2015), with a focus on coral records that allow not only for hydroclimate reconstruction but also temperature. The new CoralHydro2k database will compile a comprehensive reconstruction of the $\delta^{18} \mathrm{O}$ of seawater for the tropical oceans. This requires an in-depth understanding of each coral record, including the intricacies of how the coral grew and the sampling methods. As such, CoralHydro2k is also investigating the impact of these methodological influences on the reconstructions using the pseudo-proxy approach. During the workshop, various experts presented new coral records to be included in the database and additional approaches that have not been explored by CoralHydro2k. Subsequently, we have targeted a number of primary projects to be completed with a paper as the final deliverable:

\section{(1) Build a CoralHydro2k database}

Only a handful of century-long coral-based $\delta^{18} \mathrm{O}$ seawater reconstructions currently exist. Based on these records and a number of emerging records since the Ocean $2 \mathrm{k}$ reconstruction effort, the group has developed a database of the tiered records that fulfill criteria deemed important. The criteria for Tier 1 include time coverage (most of the 20th century), resolution (monthly to seasonal), and paired coral $\mathrm{Sr} / \mathrm{C}$ a and $\delta^{18} \mathrm{O}$ to reconstruct salinity. The other tiers relax these requirements. The database will incorporate best practices learned from previous $2 \mathrm{k}$ efforts including Iso2k (Konecky et al. 2020) and PaCTS 1.0 (Khider et al. 2019).

(2) Develop a $\delta^{18} \mathrm{O}$ of seawater reconstruction

With the CoralHydro2k database, we are developing a spatial-temporal salinity and temperature reconstruction for the tropical oceans to identify the dynamical drivers of seawater $\delta^{18} \mathrm{O}$ changes. This reconstruction of tropical hydroclimate will distinguish

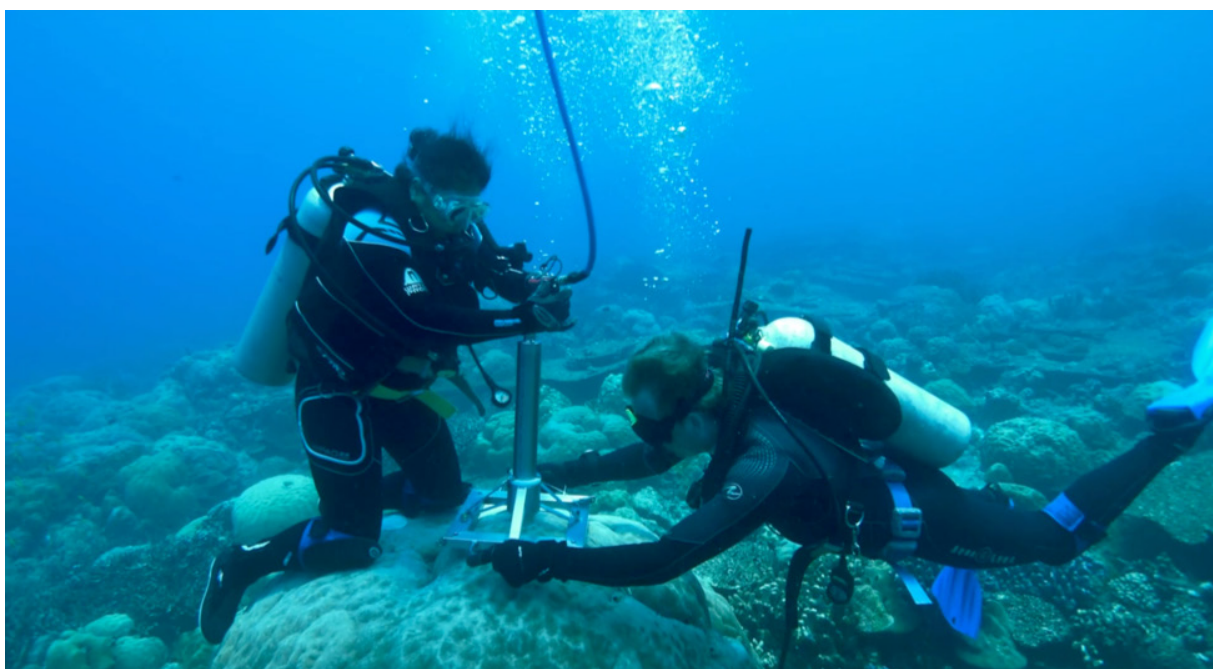

Figure 1: Extracting a core from a coral colony at Christmas Island (Indian Ocean). Photo credit: Jason Turl.

natural variability from anthropogenic trends and linkages between marine and terrestrial hydrocycles by comparing them with the observational datasets (Hakim et al. 2016) and simulations from climate models that include water-isotope tracers.

(3) Pseudo-proxy assessment of coral reconstruction methods

Use the pseudo-proxy approach to better understand how the various methods used in coral-based reconstructions impact proxy-climate calibrations and ultimately the hydroclimate interpretation. Parameters to be tested include skeletal thickening, annual average versus monthly resolution, proxy uncertainties, sea surface temperature data products, and methods used for micro-sampling, regression, and age-model development.

CoralHydro2k holds bi-weekly teleconferences that are open to anyone interested in joining the project; please contact the group leaders to get involved (pastglobalchanges. org/science/wg/2k-network/projects/ coral-hydro/people).

\section{AFFILIATIONS}

${ }^{1}$ Research School of Earth Sciences, Australian National University, Canberra, Australia ${ }^{2}$ ARC Centre of Excellence for Climate Extremes, Australian National University, Canberra, Australia ${ }^{3}$ Louisiana State University and the Coastal Studies Institute, Baton Rouge, USA

${ }^{4}$ MARUM - Center for Marine Environmental Sciences, University of Bremen, Germany

${ }^{5}$ School of Earth and Atmospheric Sciences, Georgia Institute of Technology, Atlanta, USA

\section{CONTACT}

Thomas Felis: tfelis@marum.de

REFERENCES

Felis T et al. (2018) Geophys Res Lett 45: $7112-7119$

Hakim GJ et al. (2016) J Geophys Res Atmos 121: 6745-6764

Khider D et al. (2019) Paleoceanogr Paleoclimatol 34 1570-1596

Konecky BL et al. (2020) Earth Syst Sci Data Discuss: in review, doi: 10.5194/essd-2020-5

PAGES2k Consortium (2017) Sci Data 4: 170088 Tierney JE et al. (2015) Paleoceanography 30: 226-252 